\title{
Intracystic Injection of Methotrexate in Postmenopausal Female Suffering from Simple Ovarian Cyst with Low Malignancy Index
}

\author{
Amal Abdelsamea Elsokary, Ahmed Mahoud Elkhyat and Shereef lotfy Elshwaikh* \\ Lecturer of Obstetrics and Gynecology, Faculty of Medicine, Tanta University, Egypt
}

*Corresponding author: Shereef lofty Elshwaikh, Lecturer of Obstetrics and Gynecology, Faculty of Medicine, Tanta University, Egypt.

Received Date: March 17, 2021

Published Date: March 24, 2021

\begin{abstract}
Objective: To evaluate Intracystic injection of methotrexate in postmenopausal female suffering from simple ovarian cyst with low malignancy index.

Methods: Clinical randomized controlled study, two group of post-menopausal females each group had 25 patients with ovarian cyst with low malignancy index (less than 25), group I : underwent ultrasound guided aspiration of the cyst followed by methotrexate injection Group II: had no interference. Both group will be followed up for 6 months .

Results: Epidemiological data and the characteristics of the cyst at the beginning of the study showed insignificant relationship between two groups. In group I (study group) the time of the procedure was $12.15 \pm 1.035 \mathrm{~min}$., with no complications. After follow up of the cases for 6 month it shows significant difference as regard recurrence / persistence of the cyst ( I case recurred in group 1 , and 15 case persist in group 2) with P value 0.000022 .While there was insignificant relationship as regard new symptoms, presence of complications and need for surgery .

Conclusion: This technique is considered a promising technique for its management, but it couldn't be recommended by our study as this process is invasive and it shows only single advantage over expectant management.
\end{abstract}

Keywords: Post menopause; Cyst; Malignancy index; Methotrexate

\section{Introduction}

Although ovarian cysts are more prevalent in premenopausal women, but ovarian cysts in postmenopausal women are common also, this attributed to the advances in the use of ultrasound in the screening and diagnosis of different lesions [1]. Before the era of use of ultrasound, most gynecologists were depending on palpable postmenopausal ovaries in their management, and it was considered as an indication for surgery [2].

In order to evaluate women, and exclusion of malignancy, simple and available tests are used. At present, these tests are serum CA125 measurement and ultrasound [3]. Of course, the best method for diagnosis of ovarian cancer is offered if a laparotomy and full staging procedure is carried. But, the large prevalence of benign ovarian cysts in the postmenopausal population and the increase in their diagnosis means that it would not be feasible [4].

management of postmenopausal ovarian cyst was a matter of debate for fear of malignancy. But after the advances in the diagnostic imaging technique especially ultrasound, the first step in management of postmenopausal ovarian cyst is to evaluate the lesion by transvaginal ultrasound, Doppler ultrasound could be used also but it is value is not well established, also the role of 
other imaging modalities like magnetic resonance imaging (MRI), computed tomography (CT) and positron emission tomography (PET), not also clearly established [5].

In over $80 \%$ of ovarian cancer cases had raised Serum CA125 and, if a cut-off of $30 \mathrm{u} / \mathrm{ml}$ is used, the test has a sensitivity of $81 \%$ and specificity of 75\%.5 Ultrasound is also achieving a sensitivity of $89 \%$ and specificity of $73 \%$ when using a morphology index [6].

It is recommended that a 'risk of malignancy index' should be. An effective way of triaging women into those who are at low, moderate, or high risk of malignancy and who hence may be managed by a general gynecologist, or in a cancer unit or cancer center respectively [7].

\section{Risk of Malignancy Index Compose of}

\section{Ultrasound scans: for each of the following characteristics}

(U):

- Multilocular cyst

- $\quad$ Evidence of solid areas

- $\quad$ Evidence of metastases

- $\quad$ Presence of ascites.

- $\quad$ Bilateral lesions

Each characteristic will have one point

1. $\quad U=1$ (for ultrasound score of $0-1$ ); $U=3$ (for ultrasound score of 2-5)

2. 3 points for all postmenopausal women dealt with and one point for premenopausal women (M).

3. CA125 is serum CA125 measurement in $\mathrm{u} / \mathrm{ml}$

$\mathrm{RMI}=\mathrm{U} \times \mathrm{M} \times \mathrm{CA} 125$

The women considered of low risk if RMI less than 25, and of moderate risk if RMI 25-250, and RMI will b high if more than 250 [7].

\section{Methods}

\section{Study design: randomized controlled clinical study}

\section{Setting: Tanta university hospital}

\section{Number of cases: 50 patients.}

Timing of the study: from December 1, 2017 to May 1, 2019.

Cases selection: the cases will be selected from the females whom attending Tanta university hospital,

They are selected according to the following criteria:

1. Postmenopausal female

2. Simple ovarian cyst
3. Calculated malignancy index is less than 25 ( low malignancy index)

And they are excluded if:

1. Medical disorders like liver disease or renal disease

2. Known to have allergy to methotrexate

3. Her blood picture shows low leucocytes count

4. She had previous pelvic surgery with rising suspicious of presence of dense pelvic adhesions

Sample size calculation: The sample size was calculated using Epi-Info 7 specific program. The confidence level was $90 \%$ and confidence interval was [4].

\section{Methods}

- Written consent taken from all patients submitted to the study with clarification of the methods, value and hazards of the study.

- $\quad$ Detailed history taking from all patients

- Then patients will be randomly divided into two groups by alternate randomization ( even and odd number)

\section{Group I (study group): 25 patients}

1. All patients will be examined for the exclusion of vaginal infection.

2. The patient will be positioned in lithotomy position

3. Chlorhexidine will be used for vaginal cleansing.

4. The needle used 16-gauge, $35 \mathrm{~cm}$ long, attached to transvaginal probe (Samsung Medison.UGEO H60; Korea).

5. The transvaginal transducer will be attached to needle guide introduced into the vagina

6. The needle will be introduced through needle guide; its course will be noticed in the screen. Till it seen inside the cyst. a syringe will be connected to the needle

7. Aspiration of the content of the cyst will be done using negative pressure and the cyst will be completely evacuated.

8. Cyst contents will be sent for bacteriological and cytological examination.

9. While the needle is inside the cyst , $3 \mathrm{ml}$ of normal saline was added to one ampoule of methotrexate $50 \mathrm{mg}$ and then injected inside cyst cavity under ultrasonic guidance

10. Postoperative follow up of patient for pain and fever for 24 hours, then ultrasound will be done to the patient before discharge, and appropriate antibiotic will be given to the patient.

11. Follow up of the patient will be done for 6 months interval for the recurrence of the lesion and CA-125 level 


\section{Group II(control group):25 patients}

1. No interference

2. Follow up of the patient will be done for 6 months for the recurrence of the lesion and CA-125 level.

\section{Outcome}

All patients in both groups will be assessed and compared according to the following outcomes:

- $\quad$ Recurrence of the cyst

- $\quad$ Change in the cyst size

- $\quad$ Change in CA-125 level

- Occurrence of complications related to the cyst ( rupture , heamorrhage...)

- $\quad$ Appearance of a new symptoms

- Need of other surgical interference like exploration

- Post-operative complication : like postoperative pain , postoperative sepsis

\section{Ethical Approval and Clinical Trial Registration}

This study was approved by local ethical committee of Tanta University before the start of this study and registered on Pan African Clinical Trials Registry (pactr) under the code of PACTR201810634381311 All patients were informed about study design, interventions, and risks. All patients signed written consent. Privacy and security were maintained all over the duration of study.

There were no unexpected risks during the course of research.

\section{Results}

50 patients were selected in the course of the study fulfilling the inclusion and exclusion criteria , any dropped case during the course of study ( follow up) was excluded and replaced by another case fulfilling the same inclusion and exclusion criteria.

Studying of epidemiological data showed insignificant relationship between two groups as regards the age of patient, duration of menopause, gravidity, parity and BMI. (Table 1).

Studying of the characteristics of the cyst at the beginning of the study showed insignificant relationship between two groups as regards size of the cyst, CA-125 level and malignancy index (Table 1).

In group I (study group) 25 patients underwent cyst aspiration, the time of the procedure from the beginning of application of antiseptic solution to the end of aspiration was $12.15 \pm 1.035 \mathrm{~min}$. (range 9-18 min) (Table 1). From all 25 patients five patients had postoperative complications in the form of post aspiration dull aching lower abdominal pain which was managed by post aspiration parentaral NSAIDs drug injection (Table 2). The result of bacteriological and cytological examination of the sent aspirated fluid revealed no malignant cells and no bacteriological growth.

Table1: shows distribution of cases according demographic data and the characteristics of cyst at the beginning of the study.

\begin{tabular}{|c|c|c|c|c|c|c|}
\hline & \multicolumn{2}{|c|}{ Group I (Study Group) } & \multicolumn{2}{|c|}{ Group II (Control Group) } & \multicolumn{2}{|c|}{ Chi-square } \\
\hline & Range & Mean \pm S. D & Range & Mean \pm S. D & T value & $P$ value \\
\hline Age & $47-60$ & $52.04 \pm 4.098$ & $48-63$ & $52.6 \pm 4.21$ & -0.476 & 0.317 \\
\hline Duration of menopause & $1-11$ & $3.72 \pm 2.59$ & $1-11$ & $4.04 \pm 2.79$ & -0.42 & 0.338 \\
\hline Gravidity & $1-7$ & $4.12 \pm 1.786$ & $2-8$ & $4.4 \pm 1.802$ & -0.551 & 0.291 \\
\hline Parity & $1-6$ & $3.24 \pm 1.392$ & $1-6$ & $3.4 \pm 1.322$ & -0.416 & 0.339 \\
\hline BMI & $23-32$ & $28.28 \pm 2.354$ & $25-32$ & $28.68 \pm 2.055$ & -0.639 & 0.262 \\
\hline Size of cyst & $3-8$ & $5.12 \pm 1.12$ & $3-7$ & $4.6 \pm 1.08$ & 1.66 & 0.051 \\
\hline Pre CA-125 & $4.5-8.2$ & $6.904 \pm 1.011$ & $4.2-8.2$ & $6.528 \pm 1.26$ & 1.163 & 0.125 \\
\hline Post CA-125 & $7.8-3.3$ & $6.196 \pm 1.033$ & $7.9-3.9$ & $5.964 \pm 1.283$ & & \\
\hline MI & $13.5-24.6$ & $20.712 \pm 3.035$ & $12.6-24.6$ & $19.584 \pm 3.78$ & 1.163 & 0.125 \\
\hline Change size of cyst & 2 & $2 \pm N / A$ & $1-3$ & $2.2 \pm 1.78$ & $\mathrm{~N} / \mathrm{A}$ & $\mathrm{N} / \mathrm{A}$ \\
\hline Time of aspiration & $9-18$ & $12.15 \pm 1.035$ & $\mathrm{~N} / \mathrm{A}$ & $\mathrm{N} / \mathrm{A}$ & $\mathrm{N} / \mathrm{A}$ & $\mathrm{N} / \mathrm{A}$ \\
\hline
\end{tabular}

Table 2: shows cyst and patient data after 6 months follow up.

\begin{tabular}{|c|c|c|c|c|c|c|}
\hline & \multicolumn{2}{|c|}{ Group I } & \multicolumn{4}{|c|}{ Group II } \\
\hline & No & Yes & No & Yes & Chi-square & $P$ value \\
\hline Recurrence/ persistence & 24 & 1 & 10 & 15 & 18.01 & 0.000022 \\
\hline New symptoms & 25 & 0 & 23 & 2 & $\mathrm{~N} / \mathrm{A}$ & $\mathrm{N} / \mathrm{A}$ \\
\hline Operative complications & 20 & 5 & $\mathrm{~N} / \mathrm{A}$ & $\mathrm{N} / \mathrm{A}$ & $\mathrm{N} / \mathrm{A}$ & $\mathrm{N} / \mathrm{A}$ \\
\hline Complications & 25 & 0 & 25 & 0 & $\mathrm{~N} / \mathrm{A}$ & $\mathrm{N} / \mathrm{A}$ \\
\hline Need for surgery & 25 & 0 & 25 & 0 & $\mathrm{~N} / \mathrm{A}$ & $\mathrm{N} / \mathrm{A}$ \\
\hline
\end{tabular}


After follow up of the cases in both group for six month it shows significant difference as regard recurrence / persistence of the cyst ( I case recurred in group1 and 15 case persist in group 2) with P value 0.000022 (Table 2), the only case which recurred in group1, the cyst size after recurrence was even smaller $(4 \mathrm{~cm}$ before aspiration, $2 \mathrm{~m}$ in recurrence after 6 month), while 15 case which persist in group2, 5 case persist in the same size , three cases decrease in size, and seven case the cyst increased in size ( increase range from $1-3 \mathrm{~cm}$ with mean $2.2 \mathrm{~cm}$ ) (Table 1 ).

While there was insignificant difference between both group as regard new symptoms, presence of complications and need for surgery , actually no cases in both group needed surgery , no cases developed cyst complications, and no cases in group I developed new symptoms, and only 2 cases in group II developed new symptoms in the form of dull aching lower abdominal pain. (Table 2).

Also, there was insignificant difference as regards CA-125 level between both group after 6 month follow up, and when we compared level of CA-125 in each group before and after 6 month follow up, we noticed no significant changes.

\section{Discussion}

The management of postmenopausal ovarian cyst was a matter of debate for fear of malignancy. But after the advances in the diagnostic imaging field especially ultrasound and CA-125 It is recommended that a 'risk of malignancy index' should be. An effective way of triaging women into those who are at low, moderate, or high risk of malignancy. The women considered of low risk if RMI less than 25 , and of moderate risk if RMI 25-250, and RMI will be high if more than 250 [7].

In our study we had two group of post menopause patients each group had 25 patients with ovarian cyst with low malignancy index, group I : underwent ultrasound guided aspiration of the cyst followed by methotrexate injection. Group II : had no interference except for follow up of the cyst.

Results showed that in group I (study group) the time of the procedure was $12.15 \pm 1.035 \mathrm{~min}$. (range 9-18 min) (Table 1). With 5 patients had postoperative abdominal pain (Table 2). After follow up of the cases in both group for 6 month it showed significant relationship as regard recurrence / persistence of the cyst (I case recurred in group1, and 15 case persist in group 2) with $P$ value 0.000022 (Table 2).While there was insignificant difference between both group as regard new symptoms, change of CA-125 level, presence of complications and need for surgery.

Our study agreed with Ewa K, et al. [8] who followed up 40 postmenopausal female with small, anechoic, thin-walled ovarian cysts for 9 years, they found that, 38\% of cases the cyst completely disappear( $40 \%$ in our study), $59 \%$ persist ( $60 \%$ in our study) , but $3 \%$ of cases the cyst increase in size ( 0 case increase in size in follow up group in our study and this may attributed to the short duration of follow up of cases ,6 months, in our study ) , and they recommended follow up for postmenopausal ovarian simple cyst with low malignancy index [8].

Our study agreed with Auslender R, et al. [9] who followed up 51 postmenopausal patients with small $(<5 \mathrm{~cm})$, smooth, aseptate, clear ovarian cysts, without ascites, by vaginal ultrasound examinations and CA-125 level for a period of 2.5 years. And they found that none of the cysts showed changes in texture, nor did ascites appear, the mean size of the cysts decreased with time and the CA-125 antigen serum levels remained low. And they recommend conservative follow-up of small, simple cysts in postmenopausal women [9].

Spyros M, et al. [10] had evaluated ultrasonography guided cyst aspiration and methotrexate injection in the management of simple and Endometriotic ovarian cysts over 162 females with cyst size (3$10.6 \mathrm{~cm}$ ) and follow up for 6 months , the cyst disappears in $83.8 \%$ of cases [10]. Also, RN Troiano, et al. [12] and Pratiksha G, et al. [12] who had a similar study over 132 patients with success rate 90.9\% with no major complications were observed in both studies ,despite that the age group in previous studies study (15-77 years, 15-72 years) differ from our study but as the efficacy of aspiration and methotrexate injection was promising in that study, which may support our study when we choose specific age group with success rate reach $97 \%$ [12].

Luciano GN et al. [13] submitted a retrospective observational study , 226 postmenopausal female with unilocular ovarian cysts less than $50 \mathrm{~mm}$ in diameter and with normal serum CA 125 levels with the duration of follow up was 5 years, they found that $11.1 \%$ of cases had increased cyst size and raised serum CA-125 level and so suggested the expectant management of those female, if compared with our study no case had an increase in size of the cyst in follow up group nor increase in CA-125 level and this may attributed to the short duration of follow up of cases 6 months [13].

Our study agreed with Josep MA et al. [14] who evaluated the expectant management of asymptomatic, simple ovarian cysts with size (from 1.5 to $5.0 \mathrm{~cm}$ ) diagnosed by ultrasound in 36 postmenopausal women, for average 31.5 months. They found that no cases of cyst enlargement. The cysts remained unchanged in (80.5\%), decreased in size in (11.1\%) however the cyst disappeared in $(8.3 \%)$, it was $40 \%$ in control group our study, and it may attributed to the criteria of choosing females with low malignancy index based on low level of CA-125 which was not measured in their study [14].

Our study agreed with Madhutandra S, et al. [15] who studied retrospectively the role of conservative management of simple ovarian cyst in postmenopausal female from 1997 to 2010 over 314 females, they found that (46.30\%) had spontaneous resolution and (43.91\%) persisted unchanged over the follow-up period. (1.06\%) significantly increased in size. Interestingly these numbers were very similar to the numbers of control group in our study [15]. 
M Nikolaou, et al. [16] had evaluated the efficacy of transvaginal ultrasound-guided aspiration of benign ovarian cysts without injection of any substance on 46 women all women were reevaluated at 1, 3 and 6 months after the procedure. The cytological analysis was negative for malignancy in all cases. They found that the overall recurrence rate for ovarian cysts of $39.1 \%$. If compared to our study, we had a higher success rate with the recurrence only $3 \%$, it is due to the injection of locally acting methotrxate after aspiration of the cyst which would decrease the recurrence rate significantly [16].

Marta C, et al. [17] had compared the efficacy of ultrasoundguided aspiration versus aspiration with ethanol sclerotherapy in the management of simple adnexal cysts measuring 3 to $10 \mathrm{~cm}$. despite the use of another material over 75 women; they had similar results to our study in the injection group with lower recurrence rate in postmenopausal female (9\%). In our study it was $3 \%$ and this slight difference may be due to use of another material [17].

We concluded that Intracystic methotrexate injection in postmenopausal female suffering from ovarian cyst with low malignancy index is considered a promising technique for its management, but it couldn't be recommended by our study as this process is invasive (the patients had good compliance though) and it shows only single advantage over expectant management as regards the persistence/recurrence rate with no other advantage within the six month duration of follow up I our study.

\section{Acknowledgement}

None.

\section{Conflict of interest}

Authors declare no conflicts of interest.

\section{References}

1. Greenlee RT, Kessel B, Williams CR, Riley TL, Ragard LR, et al. (2010) Prevalence, incidence, and natural history of simple ovarian cysts among women $>55$ years old in a large cancer screening trial. Am J Obstet Gynecol 202(4): 373-379.

2. Zalud I, Busse R, Kurjak BF (2013) Asymptomatic simple ovarian cyst in postmenopausal women: syndrome of 'visible ovary'. Donald School Ultrasound Obstet Gynecol 7: 182-186.

3. Zurawski VR Jr, Orjaseter H, Andersen A, Jellum E (1988) Elevated serum CA 125 levels prior to diagnosis of ovarian neoplasia: relevance for early detection of ovarian cancer. Int J Cancer 42(5): 677-680.
4. Yuen PM, Yu KM, Yip SK, Lau WC, Rogers MS, et al. (1997) A randomized prospective study of laparoscopy and laparotomy in the management of benign ovarian masses. Am J Obstet Gynecol 177(1): 109-114.

5. Brown DL, Doubilet PM, Miller FH, Frates MC, Laing FC,et al. (1998) Benign and malignant ovarian masses: selection of the most discriminating gray-scale and Doppler sonographic features. Radiology 208(1): 103-110

6. Tuxen MK, Sölétormos G, Petersen PH, Schiøler V, Dombernowsky P (1999) Assessment of biological variation and analytical imprecision of CA 125, CEA, and TPA in relation to monitoring of ovarian cancer. Gynecol Oncol 74(1): 12-22.

7. (2011) RCOG, Royal College of Obstetricians and Gynaecologists. Management of Suspected Ovarian Masses in Premenopausal Women. Guideline no. 62 .

8. Ewa K, Ellika A (1995) Diagnosis and follow-up of simple ovarian cysts detected by ultrasound in postmenopausal women. Obstet Gynecol 85(2): 211-214.

9. Auslender R, Atlas I, Lissak A, Bornstein J, Atad J, et al. (1996) Follow up of small, postmenopausal ovarian cysts using vaginal ultrasound and CA-125 antigen. J Clin Ultrasound 24(4): 175-178.

10. Spyros M, George D, Athanasios P, Nikolaos P, Nikolaos Th, et al. (2005) Management of Ovarian Cysts with Aspiration and Methotrexate Injection. Radiology 235(2): 748-751.

11. Troiano RN, Taylor KJ (1998) Sonographically guided therapeutic aspiration of benign-appearing ovarian cysts and endometriomas. American Journal of Roentgenology 171(6): 1601-1605.

12. Pratiksha G, Anju H (2016) Management of ovarian cysts with percutaneous aspiration and methotrexate injection. Niger Med J 57(1): $19-23$

13. Luciano GN, Nicolette DK, Philip WR (2003) Persistent unilocular ovarian cysts in a general population of postmenopausal women: is there a place for expectant management? Obstet Gynecol 102(3): 589-593.

14. Josep MA, Cristobal R, Argacha P, Romero F, Leira J, et al. (1998) Simple adnexal cysts in postmenopausal women: conservative management. Maturitas 30(1): 51-54

15. Madhutandra S, Mark GW (2012) Simple ovarian cysts in postmenopausal women: scope of conservative management. European Journal of Obstetrics \& Gynecology and Reproductive Biology 162(1): 75-78.

16. Nikolaou M, Adonakis G, Zyli P, Androutsopoulos G, Saltamavros A, et al. (2014) Transvaginal ultrasound-guided aspiration of benign ovarian cysts. J Obstet Gynaecol 34(4): 332-335.

17. Marta C, Amparo GT, Ramon C, Enric C, Jordi P (2016) Ultrasound-guided aspiration with and without ethanol sclerotherapy in the management of simple adnexal cysts: a single-center experience. J Minim Invasive Gynecol 23(2): 242-251. 